\title{
Solubility of palladium in picritic melts: 1 . The effect of iron
}

\author{
V. Laurenz ${ }^{\mathrm{a}, *}$, R.O.C. Fonseca ${ }^{\mathrm{a}}$, C. Ballhaus ${ }^{\mathrm{a}}$, P.J. Sylvester ${ }^{\mathrm{b}}$ \\ a Steinmann Institut für Geologie, Mineralogie und Paläontologie, Rheinische Friedrich-Wilhelms-Universität Bonn, \\ Poppelsdorfer Schloss, 53115 Bonn, Germany \\ ${ }^{\mathrm{b}}$ Department of Earth Sciences, Memorial University of Newfoundland, 300 Prince Philip Drive, St. John's, Newfoundland, Nfld, \\ Canada A1B $3 \times 5$
}

Received 4 April 2009; accepted in revised form 11 February 2010; available online 23 February 2010

\begin{abstract}
In order to improve our understanding of HSE geochemistry, we evaluate the effect of Fe on the solubility of Pd in silicate melts. To date, experimentally determined Pd solubilities in silicate melt are only available for Fe-free anorthite-diopside eutectic compositions. Here we report experiments to study the solubility of $\mathrm{Pd}$ in a natural picritic melt as a function of $\mathrm{pO}_{2}$ at $1300^{\circ} \mathrm{C}$ in a one atm furnace. Palladium concentrations in the run products were determined by laser-ablationICP-MS. Palladium increases from $1.07 \pm 0.26 \mathrm{ppm}$ at FMQ-2, to $306 \pm 19 \mathrm{ppm}$ at FMQ+6.6. At a relative $\mathrm{pO}_{2}$ of FMQ the slope in $\log \mathrm{Pd}$ concentration vs. $\log \mathrm{pO}_{2}$ space increases considerably, and Pd concentrations are elevated over those established for AnDi melt compositions. In the same $\mathrm{pO}_{2}$ range, ferric iron significantly increases relative to ferrous iron. Furthermore, at constant $\mathrm{pO}_{2}(\mathrm{FMQ}+0.5) \mathrm{Pd}$ concentrations significantly increase with increasing $\mathrm{X}_{\mathrm{FeO}-\text { total }}$ in the melt. Therefore, we consider ferric Fe to promote the formation of $\mathrm{Pd}^{2+}$ enhancing the solubility of $\mathrm{Pd}$ in the picrite melt significantly.

The presence of $\mathrm{FeO}$ in the silicate melt has proven to be an important melt compositional parameter, and should be included and systematically investigated in future experimental studies, since most natural compositions have substantial $\mathrm{FeO}$ contents.
\end{abstract}

(C) 2010 Elsevier Ltd. All rights reserved.

\section{INTRODUCTION}

The highly siderophile elements (HSE - Pt, Pd, Rh, Os, $\mathrm{Ir}, \mathrm{Ru}, \mathrm{Re}$ and $\mathrm{Au}$ ) are among the rarest and most valuable elements found on Earth. Not only are they useful in tracing processes such as core-mantle differentiation (e.g. Kimura et al., 1974; Chou, 1978; O'Neill, 1991; Righter et al., 2008) and possible core-mantle interaction (e.g. Brandon et al., 1999; Brandon and Walker, 2005; Puchtel et al., 2005); they are also key to understanding processes within the Earth's mantle, including partial silicate melting (e.g. Barnes et al., 1985; Philipp et al., 2001; Pearson et al., 2004), melt segregation (Bockrath et al., 2004), and mantle metasomatism (e.g. Becker et al., 2001; Alard et al., 2005; Lorand et al., 2008; Luguet et al., 2008).

\footnotetext{
* Corresponding author. Tel.: +49 0228733022.

E-mail address: laurenz@uni-bonn.de (V. Laurenz).
}

The primary reservoir for the HSE is the Earth's mantle. Estimates of total HSE in primitive upper mantle (PUM) range from 23 to $32 \mathrm{ppb}$ (e.g. Palme and O'Neill, 2003; Becker et al., 2006). The transport of the HSE from the mantle to the crust is via primitive silicate mantle melts, either as oxide and sulfide species dissolved in silicate melt (e.g. Borisov and Palme, 2000) or as HSE-bearing sulfide melt droplets in suspension in a silicate melt (Bockrath et al., 2004; Ballhaus et al., 2006; Fonseca et al., 2009). In order to discriminate between these transport modes, it is essential to evaluate how and to what extent the HSE are dissolved in silicate melt.

Previous experimental studies (Capobianco et al., 1993; Borisov et al., 1994, 2004; Borisov and Palme, 1995, 1997, 2000; O'Neill et al., 1995; Capobianco and Hervig, 1996; Ertel et al., 1999, 2001, 2008; Fortenfant et al., 2003, 2006; Cottrell and Walker, 2006; Righter et al., 2008; Yokoyama et al., 2009) have shown that the HSE are dissolved in silicate melts as oxide species, according to 
$M^{\text {alloy }}+\frac{x}{4} \mathrm{O}_{2} \Longleftrightarrow \mathrm{MO}_{x / 2}^{\text {silicate }}$

where $x$ is the valence state of the metal $M$ in the silicate melt. One of the principal variables in controlling HSE solubility is the oxygen partial pressure $\left(\mathrm{pO}_{2}\right)$. The activity of the HSE oxide species in solution in silicate $\left(a_{\mathrm{MO}_{x / 2}}^{\text {silicate }}\right)$ is given by

$\log a_{\mathrm{MO}_{x / 2}}^{\text {silicate }}=\log K+\log a_{M}^{\text {alloy }}+\frac{x}{4} \log \mathrm{pO}_{2}$

and may be calculated if the equilibrium constant $K$ and the activity-composition relations for both metal and oxide species in solution are known. Assuming that HSE dissolution in silicate melt is ideal, the activity coefficient $\left(\gamma_{\mathrm{MO}_{x / 2}}^{\text {silicate }}\right)$ is unity. For constant melt compositions the valence state of the HSE cationic species can then be derived from the slope (m) of the concentration profile in log-concentration vs. $\log$ $\mathrm{pO}_{2}$ space, where the valence is given by

$m=\frac{x}{4}$

Solubilities of the HSE in silicate melts at one atm have mostly been determined in synthetic anorthite-diopside (AnDi) eutectic melt compositions (e.g. Borisov et al., 1994; O'Neill et al., 1995). However, many authors regard this system to be too simplistic (e.g. Hillgren et al., 1996; O'Neill and Eggins, 2002; Rose-Weston et al., 2009) and melt composition (i.e. $\mathrm{CaO}, \mathrm{MgO}, \mathrm{FeO}$ or $\mathrm{S}$ concentrations, degree of polymerization) has been shown to be an important factor for the solubility of trace elements in silicate melts at sulfide-undersaturated conditions. This raises the question if, and to which extent, melt components such as $\mathrm{FeO}$ and $\mathrm{S}^{2-}$ also influence HSE solubility.

Borisov and Palme (2000) considered the effect of Fe in the metal phase on HSE solubility in silicate melts by applying the activity-composition relations for Fe-HSE alloy systems to calculate the concentrations expected in natural Fe-bearing magmas. These authors stated that metallic Fe alloying with Pd will lower Pd concentration in AnDi melts, however, they downplayed possible interactions of ferrous and ferric iron species with Pd oxide species within the silicate melt. Very little experiments are available to judge if dissolved $\mathrm{S}^{2-}$ affects the solubility of HSE; however, O'Neill et al. (1995) argued that the effect must be small because the $\mathrm{S}^{2-}$ anionic ligand is about 200 times less abundant in a basaltic silicate melt than the $\mathrm{O}^{2-}$ ligand.

In this contribution, we investigate the effect of $\mathrm{FeO}$ and $\mathrm{FeO}_{1.5}$ on Pd solubility in silicate melt. We report experiments with $\mathrm{FeO}$-bearing picritic melt in $\mathrm{Pd}$ wire-loops over a range in relative $\mathrm{pO}_{2}$ from $\mathrm{FMQ}-2$ to $\mathrm{FMQ}+6.6$ (FMQ = fayalite-magnetite-quartz buffer, as calibrated by O'Neill, 1987). All Pd concentrations in silicate were measured by Laser Ablation Inductively Coupled Plasma Mass Spectrometry (LA-ICP-MS). Owing to its spatial resolution LA-ICP-MS allows the distinction between HSE in solution (cf. Eq. (1)) and HSE present as micro-nuggets, unlike Instrumental Neutron Activation Analysis (INAA) which is a bulk analytical technique (cf. Ertel et al., 2006; Ertel et al., 2008). We show that iron-oxide species play a major role in controlling the solubility of Pd in silicate melt. Not only does Fe dilute the activity of $\mathrm{Pd}$ in the $\mathrm{Pd}-\mathrm{Fe}$ alloy phase as predicted by
Borisov and Palme (2000), $\mathrm{FeO}$ and $\mathrm{FeO}_{1.5}$ species in the silicate melt undergo redox exchange reactions with dissolved $\mathrm{PdO}_{x / 2}$ species, tending to raise $\mathrm{Pd}$ solubility in silicate relative to iron oxide-free silicate melts.

\section{EXPERIMENTAL PROCEDURES}

Solubility experiments were carried out in a one atm vertical muffle tube furnace equipped for gas mixing. The starting silicate melt composition is a natural picritic basalt with $\sim 12$ wt. $\% \mathrm{MgO}$, henceforth picrite, from the Solomon Islands (SE1 - Schuth et al., 2004; Rohrbach et al., 2005). We employed the wire-loop technique where the picrite powder is placed in a $\mathrm{Pd}$ wire-loop, suspended in the hot zone of the furnace, and equilibrated at $1300{ }^{\circ} \mathrm{C}$ with a $\mathrm{CO}-\mathrm{CO}_{2}\left( \pm \mathrm{O}_{2}\right)$ gas atmosphere (cf. Fig. 1). After a run time of $48 \mathrm{~h}$, experiments were quenched in air by quickly drawing the Pd loop to the water-cooled top of the furnace which is at $\sim 100^{\circ} \mathrm{C}$. Selected experiments were reversed by equilibrating initially Pd-free picrite and picrite doped with $\mathrm{PdO}$ in the thousands of ppm concentration range, simultaneously in the same atmosphere. Temperature $\left(1300 \pm 2{ }^{\circ} \mathrm{C}\right)$ was controlled by a Type B thermocouple $\left(\mathrm{Pt}_{94} \mathrm{Rh}_{6}-\mathrm{Pt}_{70} \mathrm{Rh}_{30}\right)$ and calibrated against the melting points of $\mathrm{Au}$ and $\mathrm{Cu}$.

Oxygen partial pressure was imposed by $\mathrm{CO}-\mathrm{CO}_{2}\left( \pm \mathrm{O}_{2}\right)$ gas mixtures (Chase, 1998). Thermodynamically calculated $\mathrm{O}_{2}$ partial pressures were checked with a $\mathrm{CaO}-\mathrm{Y}_{2} \mathrm{O}_{3}$-stabilized $\mathrm{ZrO}_{2}$ solid electrolyte cell and were found to be accurate to within 0.2 log-bars of the calculated $\mathrm{pO}_{2}$ values. Above a relative $\mathrm{pO}_{2}$ of $\mathrm{FMQ}+1$, however, precision deteriorates because the amount of $\mathrm{CO}$ added to the gas becomes too small to be controlled precisely with our gas flow meters. This problem is circumvented by adding $\mathrm{O}_{2}$ to the reactant gas. Because $\mathrm{O}_{2}$ reacts with $\mathrm{CO}$ to form $\mathrm{CO}_{2}$, this technique allows $\mathrm{CO}$ flow rates well inside the cal-

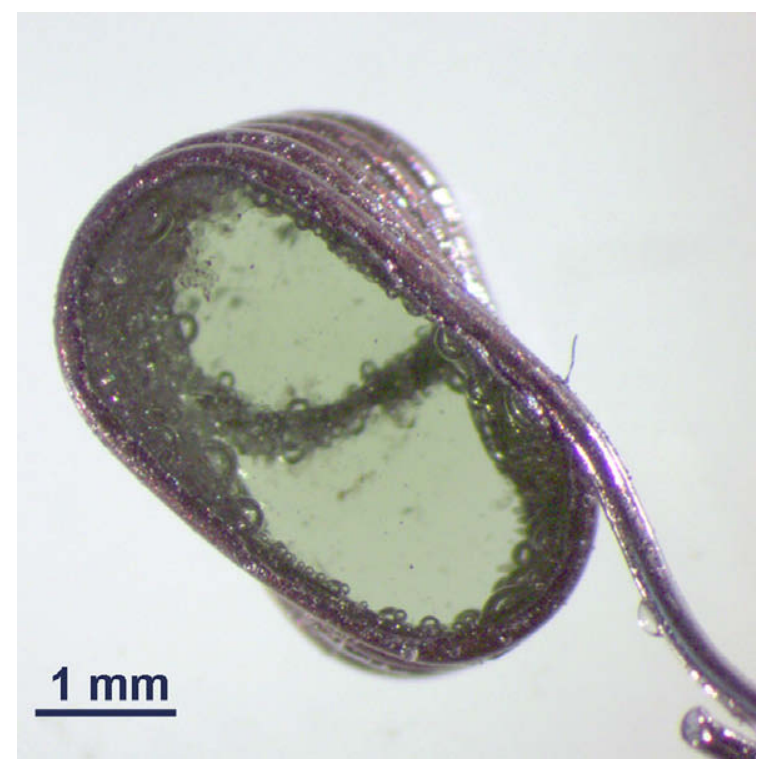

Fig. 1. Example of a quenched loop experiment. Picrite quenched to a clear green glass and attached to the Pd-wire loop. 
ibration range of the $\mathrm{CO}$ flow meter, without actually increasing pCO in the gas mix. With this technique, we can comfortably access $\mathrm{pO}_{2}$ regions at $1300{ }^{\circ} \mathrm{C}$ as high as $\mathrm{FMQ}+5$ (cf. Fig. 2). A drawback is that $\mathrm{pO}_{2}$ can no longer be calculated thermodynamically because if the variable to be calculated is added as a component, the system is overdetermined. However, as $\mathrm{pO}_{2}$ can be measured independently with a solid electrolyte cell, this is not an issue.

\section{ANALYTICAL PROCEDURES}

Experimental run products (wire loop and the silicate glass) were analyzed for their major element compositions with a JEOL JXA 8900 electron microprobe analyzer (EMPA) in wavelength dispersive mode (WDS) with an accelerating voltage of $15 \mathrm{kV}$ and a beam current of $15 \mathrm{nA}$. Standards used were natural silicates and oxides for glass analysis, and pure $\mathrm{Pd}$ and $\mathrm{Fe}$ for the analysis of the metals. Glasses were analyzed with the beam defocused to $10 \mu \mathrm{m}$ and metals with a focused beam. Counting times were $10 \mathrm{~s}$ on peak and $5 \mathrm{~s}$ on background, except for $\mathrm{Na}$ and $\mathrm{K}$, which were measured with 5 and $2.5 \mathrm{~s}$, respectively in order to avoid alkali migration. Matrix corrections were done with the ZAF correction method. To check for homogeneity we analyzed up to 15 points randomly distributed on each glass and metal. No inhomogeneities were detected. Average compositions are compiled in Table 1.

Palladium concentrations for the silicate glass charges were determined at the Inco Innovation Centre, Memorial University (MUN, New Foundland) using a GeoLas Pro laser ablation system coupled with a Finnigan Element XR inductively coupled plasmamass spectrometer. The GeoLas system consists of a Lambda Physik COMPexPro 110 ArF excimer laser operating at a wavelength of $193 \mathrm{~nm}$ and a pulse width of $20 \mathrm{~ns}$, a beam delivery and ablation cell apparatus, a camera for sample viewing and dedicated

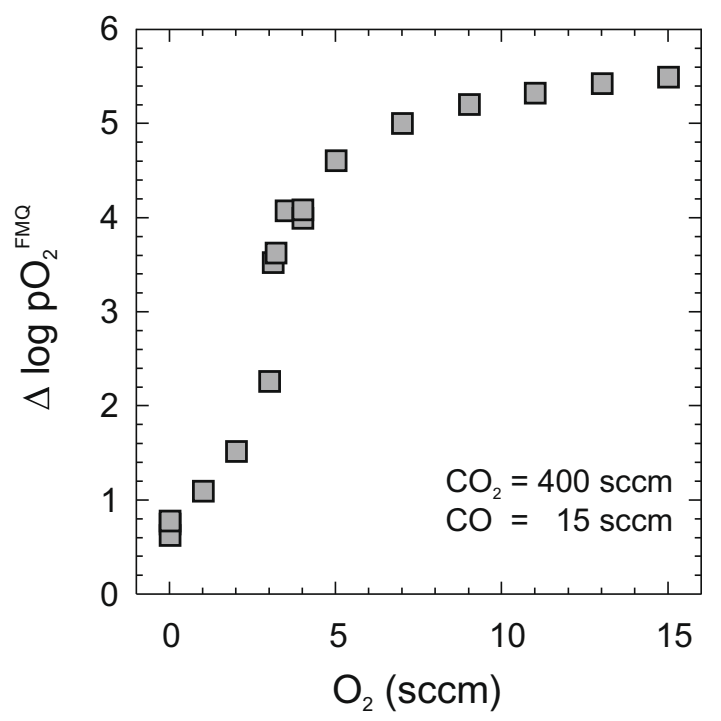

Fig. 2. Variation in relative $\mathrm{pO}_{2}$ imposed by the addition of increasing amounts of $\mathrm{O}_{2}$ to a $\mathrm{CO}-\mathrm{CO}_{2}$ gas mixture. Flow rates of $\mathrm{CO}$ and $\mathrm{CO}_{2}$ held constant at 15 and $400 \mathrm{sccm}$ (standard cubic centimeters per minute), respectively. Oxygen partial pressures were measured in situ with an Y-stabilized Zirconia cell and are given relative to FMQ (O'Neill, 1987). Errors are smaller than symbol size. Note that the measured $\mathrm{pO}_{2}$ does not match the $\mathrm{pO}_{2}$ calculated according to the NIST-JANAF tables, for reasons not evaluated yet. software for controlling ablation parameters. Polished samples were placed in an air tight chamber and ablated in helium gas, which increases analyte sensitivities by enhancing sample transport from the ablation cell during deep ultraviolet ablation. Analyses were made on a 40-micron spot with a laser fluence of $5 \mathrm{~J} / \mathrm{cm}^{2}$ and repetition rate of $10 \mathrm{~Hz}$. Data were collected in time-resolved mode with $30 \mathrm{~s}$ measurement of the pure gas background, followed by $60 \mathrm{~s}$ of measurement during laser ablation. Count rates were measured using one point per peak for each of the following isotopes: ${ }^{25} \mathrm{Mg},{ }^{27} \mathrm{Al},{ }^{29} \mathrm{Si},{ }^{43} \mathrm{Ca}$ (used for internal standardization with Ca values by EPMA), ${ }^{44} \mathrm{Ca},{ }^{65} \mathrm{Cu},{ }^{66} \mathrm{Zn},{ }^{68} \mathrm{Zn},{ }^{89} \mathrm{Y},{ }^{92} \mathrm{Zr},{ }^{105} \mathrm{Pd}$, ${ }^{106} \mathrm{Pd},{ }^{108} \mathrm{Pd}$, and ${ }^{111} \mathrm{Cd}$. Count rates for ${ }^{65} \mathrm{Cu},{ }^{66} \mathrm{Zn},{ }^{68} \mathrm{Zn},{ }^{89} \mathrm{Y}$, ${ }^{92} \mathrm{Zr}$, and ${ }^{111} \mathrm{Cd}$ were monitored for potential isobaric interferences of ${ }^{40} \mathrm{Ar}{ }^{65} \mathrm{Cu}$ and ${ }^{89} \mathrm{Y}^{16} \mathrm{O}$ on ${ }^{105} \mathrm{Pd} ;{ }^{40} \mathrm{Ar}{ }^{66} \mathrm{Zn},{ }^{90} \mathrm{Zr}{ }^{16} \mathrm{O}$ and ${ }^{106} \mathrm{Cd}$ on ${ }^{106} \mathrm{Pd}$; and ${ }^{40} \mathrm{Ar}{ }^{68} \mathrm{Zn},{ }^{92} \mathrm{Zr}^{16} \mathrm{O}$ and ${ }^{108} \mathrm{Cd}$ on ${ }^{108} \mathrm{Pd}$; but were found to be negligible in all cases.

Raw data were reduced using the macro-based spreadsheet program LAMTRACE (written by S.E. Jackson; cf. Van Achterbergh et al., 2001) for gas background subtraction, ablation interval selection and concentration calculation. In rare cases where the timeresolved data showed spikes in Pd signal, presumably due to the presence of metal nano-nuggets in the silicate glass, ablation intervals showing no spikes were selected for concentration calculations. A detailed description is given by Ertel et al. (2006). By carefully choosing the integration limits avoiding nano-nugget influenced portions of the spectra, and due to the good spatial resolution of the method, we were able to determine real Pd solubilities.

Concentrations for Pd were calculated for each of the three measured isotopes $\left({ }^{105} \mathrm{Pd},{ }^{106} \mathrm{Pd},{ }^{108} \mathrm{Pd}\right)$ by external calibration against an in-house synthetic silicate glass standard (synthesized by Holzheid et al., 2000) with a matrix composition of $50.1 \% \mathrm{SiO}_{2}$, $14.24 \% \mathrm{Al}_{2} \mathrm{O}_{3}, 9.66 \% \mathrm{MgO}, 24.75 \% \mathrm{CaO}$ and containing $131 \mathrm{ppm}$ $\mathrm{Pd}$ (as calibrated by solution-ICPMS). The calibration standard was run in duplicate before and after a series of 7-16 of the unknowns. Each glass charge was analyzed on 3-5 spots. Reported values for each spot are averages of the results for the three Pd isotopes. NIST 612 reference material was run as an unknown throughout the experiments and the measured values for $\mathrm{Pd}$ $(1.04 \pm 0.12 \mathrm{ppm})$ compared favorably to the preferred value $(1.09 \pm 0.09$ ppm, Sylvester and Eggins, 1997).

To check that Pd metal and silicate glass were homogeneous, hence verify that equilibrium was reached, up to 15 points were randomly distributed across each polished glass shard, and line profiles were run across Pd metal wires. No major compositional inhomogeneities were detected, neither in the silicate nor in the PdFe metal alloys. Forward and reverse experiments were also found to be identical in major element composition and Pd concentration, so it is assumed that redox equilibrium was generally achieved.

\section{RESULTS}

The silicate melt quenched to glasses with a light green color at low $\mathrm{pO}_{2}$ and brownish colors above FMQ+2 (Fig. 1). Some runs at low $\mathrm{pO}_{2}$ crystallized minor olivine, as confirmed by EPMA, usually found attached to the Pd-wire loop Nuggets were not found to be a problem because analysis by LAICP-MS, however, provides means of discriminating between Pd dissolved in silicate and $\mathrm{Pd}$ in metallic nuggets (e.g. Ertel et al., 2008; Médard et al., 2010).

\subsection{Metal-silicate melt equilibria with iron}

Fig. 3 shows the redox net transfer between $\mathrm{Fe}$ in the $\mathrm{Pd}$ metal and iron-oxide species in the silicate melt. The 
Table

Summary of the experimental conditions and major elements of the glasses and metal as well as the Pd concentrations obtained by LA-ICP-MS.

\begin{tabular}{|c|c|c|c|c|c|c|c|c|c|c|c|c|}
\hline $\begin{array}{l}\text { Sample } \\
\Delta \log \mathrm{pO}_{2}^{\mathrm{FMQ}} \\
\mathrm{CO} / \mathrm{CO}_{2} / \mathrm{O}_{2}(\mathrm{sccm}) \\
\end{array}$ & $\begin{array}{l}\text { PD1 } \\
-2 \\
180 / 288 / 0 \\
\end{array}$ & $\begin{array}{l}\text { PD2 } \\
-1.4 \\
74 / 240 / 0 \\
\end{array}$ & $\begin{array}{l}\text { PD3 } \\
-1 \\
54 / 280 / 0 \\
\end{array}$ & $\begin{array}{l}\text { PD4 } \\
-0.5 \\
17 / 154 / 0\end{array}$ & $\begin{array}{l}\mathrm{PD}^{\mathrm{a}} \\
-0.5 \\
17 / 154 / 0\end{array}$ & $\begin{array}{l}\mathrm{PD}^{\mathrm{a}} \\
-1 \\
27 / 140 / 0\end{array}$ & $\begin{array}{l}\mathrm{PD}^{\mathrm{a}} \\
-1.4 \\
37 / 120 / 0\end{array}$ & $\begin{array}{l}\operatorname{PD} 8^{\mathrm{a}} \\
-2 \\
60 / 96 / 0 \\
\end{array}$ & $\begin{array}{l}\text { PD9 } \\
0.5 \\
10 / 160 / 0\end{array}$ & $\begin{array}{l}\text { PD10 } \\
0 \\
10 / 160 / 0\end{array}$ & $\begin{array}{l}\text { PD11 } \\
0.5 \\
10 / 300 / 0 \\
\end{array}$ & $\begin{array}{l}\text { PD12 } \\
1 \\
8 / 400 / 0 \\
\end{array}$ \\
\hline \multicolumn{13}{|c|}{ wt. $\%$ oxide } \\
\hline$n$ & 9 & 10 & 9 & 9 & 9 & 10 & 10 & 10 & 12 & 10 & 9 & 9 \\
\hline $\mathrm{SiO}_{2}$ & $52.9 \pm 0.6$ & $52.9 \pm 0.6$ & $52.3 \pm 0.5$ & $52.4 \pm 0.5$ & $52.5 \pm 0.6$ & $52.8 \pm 0.6$ & $53.2 \pm 0.7$ & $53.4 \pm 0.4$ & $51.4 \pm 0.5$ & $52.4 \pm 0.5$ & $51.7 \pm 0.5$ & $50.4 \pm 0.6$ \\
\hline $\mathrm{TiO}_{2}$ & $0.55 \pm 0.08$ & $0.50 \pm 0.07$ & $0.50 \pm 0.10$ & $0.48 \pm 0.08$ & $0.51 \pm 0.06$ & $0.50 \pm 0.07$ & $0.51 \pm 0.05$ & $0.49 \pm 0.07$ & $0.51 \pm 0.07$ & $0.48 \pm 0.07$ & $0.48 \pm 0.06$ & $0.46 \pm 0.11$ \\
\hline $\mathrm{Al}_{2} \mathrm{O}_{3}$ & $18.1 \pm 0.2$ & $17.9 \pm 0.3$ & $17.9 \pm 0.4$ & $17.6 \pm 0.2$ & $17.9 \pm 0.3$ & $18.1 \pm 0.2$ & $17.3 \pm 0.4$ & $17.7 \pm 0.3$ & $17.0 \pm 0.2$ & $17.4 \pm 0.4$ & $17.2 \pm 0.4$ & $16.7 \pm 0.5$ \\
\hline $\mathrm{FeO}_{\text {total }}$ & $0.34 \pm 0.14$ & $0.33 \pm 0.13$ & $1.12 \pm 0.23$ & $1.20 \pm 0.21$ & $1.15 \pm 0.27$ & $0.51 \pm 0.19$ & $0.75 \pm 0.28$ & $0.17 \pm 0.11$ & $3.59 \pm 0.25$ & $2.31 \pm 0.24$ & $3.52 \pm 0.17$ & $5.41 \pm 0.36$ \\
\hline $\mathrm{MgO}$ & $11.5 \pm 0.3$ & $11.9 \pm 0.3$ & $11.3 \pm 0.2$ & $11.5 \pm 0.3$ & $11.7 \pm 0.2$ & $11.7 \pm 0.2$ & $11.9 \pm 0.2$ & $12.1 \pm 0.3$ & $11.6 \pm 0.2$ & $11.8 \pm 0.2$ & $12.0 \pm 0.3$ & $11.9 \pm 0.2$ \\
\hline $\mathrm{CaO}$ & $13.0 \pm 0.2$ & $13.1 \pm 0.4$ & $13.1 \pm 0.2$ & $13.0 \pm 0.2$ & $13.0 \pm 0.2$ & $13.0 \pm 0.2$ & $12.8 \pm 0.4$ & $13.2 \pm 0.4$ & $12.5 \pm 0.4$ & $12.7 \pm 0.2$ & $12.6 \pm 0.5$ & $12.4 \pm 0.3$ \\
\hline $\mathrm{Na}_{2} \mathrm{O}$ & $1.49 \pm 0.14$ & $1.44 \pm 0.16$ & $1.82 \pm 0.16$ & $1.74 \pm 0.18$ & $1.73 \pm 0.15$ & $1.57 \pm 0.11$ & $1.71 \pm 0.19$ & $1.41 \pm 0.13$ & $1.86 \pm 0.17$ & $1.76 \pm 0.15$ & $1.81 \pm 0.19$ & $1.79 \pm 0.17$ \\
\hline $\mathrm{K}_{2} \mathrm{O}$ & $0.77 \pm 0.10$ & $0.74 \pm 0.08$ & $0.77 \pm 0.09$ & $0.76 \pm 0.09$ & $0.72 \pm 0.07$ & $0.72 \pm 0.13$ & $0.76 \pm 0.08$ & $0.66 \pm 0.08$ & $0.73 \pm 0.07$ & $0.73 \pm 0.05$ & $0.70 \pm 0.08$ & $0.64 \pm 0.09$ \\
\hline Total & $98.9 \pm 0.9$ & $99.0 \pm 1.2$ & $99.0 \pm 0.8$ & $99.0 \pm 0.9$ & $99.3 \pm 1.0$ & $99.1 \pm 0.5$ & $99.2 \pm 1.2$ & $99.3 \pm 0.7$ & $99.5 \pm 0.9$ & $99.7 \pm 0.7$ & $100.2 \pm 1.2$ & $99.9 \pm 0.8$ \\
\hline
\end{tabular}

$\begin{array}{lllllllllllllll}X_{\mathrm{Fe}_{2} \mathrm{O}_{3}} / X_{\mathrm{FeO}}{ }^{\mathrm{b}} & 0.022 \pm 0.000 & 0.031 \pm 0.001 & 0.040 \pm 0.001 & 0.051 \pm 0.001 & 0.050 \pm 0.001 & 0.038 \pm 0.001 & 0.032 \pm 0.001 & 0.023 \pm 0.000 & 0.086 \pm 0.002 & 0.065 \pm 0.001 & 0.085 & \pm 0.001 & 0.112 \pm 0.002\end{array}$

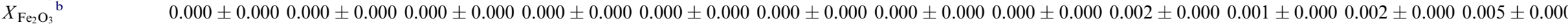

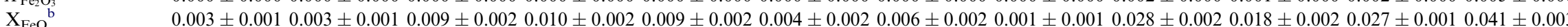

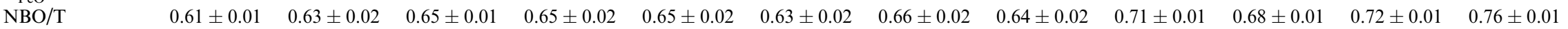

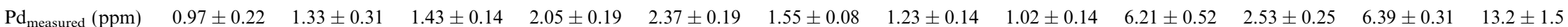

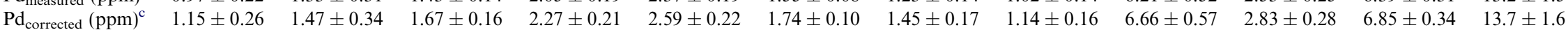

Metal:

\begin{tabular}{|c|c|c|c|c|c|c|c|c|c|c|c|c|}
\hline \multirow[b]{2}{*}{$n$} & \multicolumn{12}{|l|}{ wt. $\%$} \\
\hline & 19 & 18 & 10 & 8 & 6 & 10 & 11 & 14 & 13 & 10 & 11 & 14 \\
\hline $\mathrm{Fe}$ & $6.58 \pm 0.67$ & $4.24 \pm 0.59$ & $5.95 \pm 0.36$ & $4.28 \pm 0.42$ & $3.80 \pm 1.06$ & $4.67 \pm 0.54$ & $6.36 \pm 0.46$ & $4.59 \pm 0.37$ & $3.11 \pm 0.23$ & $4.65 \pm 0.48$ & $3.11 \pm 0.22$ & $1.90 \pm 0.21$ \\
\hline $\mathrm{Pd}$ & $93.0 \pm 0.9$ & $96.0 \pm 1.3$ & $93.5 \pm 1.0$ & $95.9 \pm 1.0$ & $95.8 \pm 1.1$ & $95.1 \pm 1.0$ & $93.6 \pm 1.6$ & $95.5 \pm 1.0$ & $96.8 \pm 1.3$ & $95.1 \pm 0.7$ & $97.3 \pm 0.7$ & $98.3 \pm 0.9$ \\
\hline Total & $99.6 \pm 0.8$ & $100.2 \pm 1.0$ & $99.5 \pm 1.1$ & $100.2 \pm 0.9$ & $99.6 \pm 0.8$ & $99.8 \pm 1.1$ & $100.0 \pm 1.6$ & $100.1 \pm 1.0$ & $99.9 \pm 1.2$ & $99.8 \pm 0.9$ & $100.4 \pm 0.7$ & $100.2 \pm 0.9$ \\
\hline$a_{\mathrm{Pd}}^{\text {alloy d }}$ & $0.84 \pm 0.02$ & $0.90 \pm 0.02$ & $0.86 \pm 0.01$ & $0.90 \pm 0.01$ & $0.91 \pm 0.02$ & $0.89 \pm 0.02$ & $0.85 \pm 0.02$ & $0.89 \pm 0.01$ & $0.93 \pm 0.02$ & $0.89 \pm 0.01$ & $0.93 \pm 0.01$ & $0.96 \pm 0.01$ \\
\hline $2 \log \left(X_{\mathrm{FeO}}^{\text {glass }} / X_{\mathrm{Fe}}^{\text {alloy }}\right)$ & $-3.24 \pm 0.36$ & $-2.91 \pm 0.36$ & $-2.14 \pm 0.19$ & $-1.81 \pm 0.17$ & $-1.76 \pm 0.32$ & $-2.63 \pm 0.33$ & $-2.53 \pm 0.33$ & $-3.54 \pm 0.58$ & $-0.62 \pm 0.08$ & $-1.33 \pm 0.13$ & $-0.64 \pm 0.08$ & $0.13 \pm 0.11$ \\
\hline
\end{tabular}




\begin{tabular}{|c|c|c|c|c|c|c|c|c|c|c|c|c|}
\hline $\begin{array}{l}\text { Sample } \\
\Delta \log \mathrm{pO}_{2} \\
\mathrm{CO} / \mathrm{CO}_{2} / \mathrm{O}_{2}\end{array}$ & $\begin{array}{l}\text { PD13 } \\
1.5 \\
5 / 500 / 0\end{array}$ & $\begin{array}{l}\text { PD14 } \\
0 \\
20 / 320 / 0\end{array}$ & $\begin{array}{l}\text { PD20 } \\
-0.5 \\
27 / 140 / 0\end{array}$ & $\begin{array}{l}\text { PD23 } \\
6.6 \\
\text { air }\end{array}$ & $\begin{array}{l}\text { PD24 } \\
4 \\
15 / 400 / 4\end{array}$ & $\begin{array}{l}\text { PD25 } \\
5 \\
15 / 400 / 7\end{array}$ & $\begin{array}{l}\text { PD30a } \\
0.5 \\
10 / 300 / 0\end{array}$ & $\begin{array}{l}\text { PD32 } \\
0.5 \\
10 / 300 / 0\end{array}$ & $\begin{array}{l}\text { PD33 } \\
0.5 \\
10 / 300 / 0\end{array}$ & $\begin{array}{l}\text { PD35 } \\
0.5 \\
10 / 300 / 0\end{array}$ & $\begin{array}{l}\text { PD36 } \\
0.5 \\
10 / 300 / 0\end{array}$ & $\begin{array}{l}\text { PD37 } \\
0.5 \\
10 / 300 / 0\end{array}$ \\
\hline \multicolumn{13}{|l|}{ Glass: } \\
\hline $\begin{array}{l}n \\
\mathrm{SiO}_{2} \\
\mathrm{TiO}_{2} \\
\mathrm{Al}_{2} \mathrm{O}_{3} \\
\mathrm{FeO}_{\text {total }} \\
\mathrm{MgO} \\
\mathrm{CaO} \\
\mathrm{Na}_{2} \mathrm{O} \\
\mathrm{K}_{2} \mathrm{O} \\
\text { Total }\end{array}$ & $\begin{array}{l}10 \\
48.7 \pm 0.7 \\
0.46 \pm 0.10 \\
16.1 \pm 0.4 \\
6.89 \pm 0.40 \\
11.3 \pm 0.1 \\
12.1 \pm 0.4 \\
2.00 \pm 0.18 \\
0.74 \pm 0.11 \\
98.5 \pm 1.3\end{array}$ & $\begin{array}{l}10 \\
52.3 \pm 0.4 \\
0.29 \pm 0.08 \\
17.5 \pm 0.4 \\
1.51 \pm 0.31 \\
12.7 \pm 0.3 \\
12.9 \pm 0.3 \\
1.72 \pm 0.17 \\
0.66 \pm 0.08 \\
99.7 \pm 0.8\end{array}$ & $\begin{array}{l}10 \\
52.5 \pm 0.6 \\
0.26 \pm 0.10 \\
17.4 \pm 0.9 \\
1.02 \pm 0.20 \\
12.6 \pm 0.3 \\
13.0 \pm 0.2 \\
1.70 \pm 0.14 \\
0.74 \pm 0.09 \\
99.4 \pm 1.5\end{array}$ & $\begin{array}{l}10 \\
48.6 \pm 0.6 \\
0.26 \pm 0.07 \\
15.9 \pm 0.4 \\
7.64 \pm 0.62 \\
12.6 \pm 0.4 \\
11.5 \pm 0.5 \\
1.95 \pm 0.14 \\
0.63 \pm 0.05 \\
99.2 \pm 1.2\end{array}$ & $\begin{array}{l}10 \\
48.4 \pm 0.8 \\
0.24 \pm 0.06 \\
16.1 \pm 0.3 \\
7.43 \pm 0.25 \\
12.7 \pm 0.3 \\
11.7 \pm 0.2 \\
2.06 \pm 0.28 \\
0.70 \pm 0.06 \\
99.5 \pm 1.1\end{array}$ & $\begin{array}{l}10 \\
48.7 \pm 0.7 \\
0.23 \pm 0.06 \\
16.1 \pm 0.3 \\
7.43 \pm 0.52 \\
12.6 \pm 0.3 \\
11.8 \pm 0.2 \\
2.05 \pm 0.09 \\
0.73 \pm 0.11 \\
99.7 \pm 0.8\end{array}$ & $\begin{array}{l}10 \\
51.9 \pm 0.4 \\
0.47 \pm 0.09 \\
17.0 \pm 0.3 \\
3.04 \pm 0.22 \\
12.4 \pm 0.2 \\
12.4 \pm 0.1 \\
1.88 \pm 0.13 \\
0.78 \pm 0.04 \\
99.9 \pm 0.7\end{array}$ & $\begin{array}{l}10 \\
48.7 \pm 0.4 \\
0.43 \pm 0.09 \\
16.0 \pm 0.2 \\
8.87 \pm 0.43 \\
11.7 \pm 0.2 \\
11.6 \pm 0.1 \\
1.62 \pm 0.13 \\
0.69 \pm 0.06 \\
99.6 \pm 1.0\end{array}$ & $\begin{array}{l}10 \\
47.0 \pm 0.6 \\
0.4 \pm 0.1 \\
15.4 \pm 0.2 \\
11.8 \pm 0.3 \\
11.2 \pm 0.2 \\
11.2 \pm 0.2 \\
1.6 \pm 0.2 \\
0.7 \pm 0.0 \\
99.3 \pm 0.8\end{array}$ & $\begin{array}{l}9 \\
40.2 \pm 0.3 \\
0.35 \pm 0.08 \\
13.1 \pm 0.2 \\
25.05 \pm 0.54 \\
9.6 \pm 0.2 \\
9.6 \pm 0.1 \\
0.86 \pm 0.11 \\
0.37 \pm 0.04 \\
99.2 \pm 0.9\end{array}$ & $\begin{array}{l}10 \\
37.5 \pm 0.3 \\
0.35 \pm 0.09 \\
11.6 \pm 0.1 \\
30.70 \pm 0.81 \\
9.0 \pm 0.2 \\
9.0 \pm 0.1 \\
0.54 \pm 0.09 \\
0.22 \pm 0.05 \\
98.9 \pm 1.3\end{array}$ & $\begin{array}{l}10 \\
43.3 \pm 0.5 \\
0.38 \pm 0.05 \\
14.1 \pm 0.2 \\
19.21 \pm 0.74 \\
10.2 \pm 0.2 \\
10.3 \pm 0.1 \\
1.12 \pm 0.13 \\
0.49 \pm 0.04 \\
99.2 \pm 1.2\end{array}$ \\
\hline $\begin{array}{l}X_{\mathrm{Fe}_{2} \mathrm{O}_{3}} / X_{\mathrm{FeO}}{ }^{\mathrm{b}} \\
X_{\mathrm{Fe}_{2} \mathrm{O}_{3}} \mathrm{~b} \\
X_{\mathrm{FeO}^{\mathrm{b}}} \\
\text { NBO/T }\end{array}$ & $\begin{array}{l}0.152 \pm 0.003 \\
0.008 \pm 0.000 \\
0.052 \pm 0.003 \\
0.78 \pm 0.02\end{array}$ & $\begin{array}{l}0.064 \pm 0.001 \\
0.001 \pm 0.000 \\
0.013 \pm 0.002 \\
0.71 \pm 0.02\end{array}$ & $\begin{array}{l}0.039 \pm 0.001 \\
0.000 \pm 0.000 \\
0.008 \pm 0.002 \\
0.70 \pm 0.03\end{array}$ & $\begin{array}{l}1.914 \pm 0.038 \\
0.043 \pm 0.003 \\
0.022 \pm 0.001 \\
0.59 \pm 0.02\end{array}$ & $\begin{array}{l}0.526 \pm 0.016 \\
0.022 \pm 0.001 \\
0.041 \pm 0.001 \\
0.74 \pm 0.02\end{array}$ & $\begin{array}{l}0.872 \pm 0.009 \\
0.029 \pm 0.002 \\
0.034 \pm 0.002 \\
0.68 \pm 0.01\end{array}$ & $\begin{array}{l}0.085 \pm 0.001 \\
0.002 \pm 0.000 \\
0.024 \pm 0.002 \\
0.73 \pm 0.02\end{array}$ & $\begin{array}{l}0.089 \pm 0.001 \\
0.006 \pm 0.000 \\
0.069 \pm 0.003 \\
0.84 \pm 0.02\end{array}$ & $\begin{array}{l}0.092 \pm 0.001 \\
0.009 \pm 0.000 \\
0.093 \pm 0.002 \\
0.90 \pm 0.02\end{array}$ & $\begin{array}{l}0.099 \pm 0.001 \\
0.020 \pm 0.000 \\
0.201 \pm 0.003 \\
1.18 \pm 0.01\end{array}$ & $\begin{array}{l}0.103 \pm 0.001 \\
0.026 \pm 0.001 \\
0.247 \pm 0.004 \\
1.35 \pm 0.02\end{array}$ & $\begin{array}{l}0.095 \pm 0.002 \\
0.015 \pm 0.001 \\
0.153 \pm 0.004 \\
1.04 \pm 0.03\end{array}$ \\
\hline $\begin{array}{l}\operatorname{Pd}_{\text {measured }}(\mathrm{ppm}) \\
\operatorname{Pd}_{\text {corrected }}(\mathrm{ppm})^{\mathrm{C}}\end{array}$ & $\begin{array}{l}19.2 \pm 2.8 \\
19.5 \pm 2.9\end{array}$ & $\begin{array}{l}2.96 \pm 0.15 \\
3.26 \pm 0.20\end{array}$ & $\begin{array}{l}1.88 \pm 0.15 \\
2.10 \pm 0.19\end{array}$ & $\begin{array}{l}307 \pm 19 \\
307 \pm 20\end{array}$ & $\begin{array}{l}70.1 \pm 7.7 \\
70.3 \pm 7.9\end{array}$ & $\begin{array}{l}114 \pm 3 \\
114 \pm 5\end{array}$ & $\begin{array}{l}5.70 \pm 0.25 \\
6.30 \pm 0.32\end{array}$ & $\begin{array}{l}5.54 \pm 0.72 \\
6.62 \pm 0.87\end{array}$ & $\begin{array}{l}5.50 \pm 0.55 \\
6.74 \pm 0.69\end{array}$ & $\begin{array}{l}7.11 \pm 0.26 \\
9.19 \pm 0.36\end{array}$ & $\begin{array}{l}7.37 \pm 0.50 \\
9.67 \pm 0.68\end{array}$ & $\begin{array}{l}5.84 \pm 0.56 \\
7.42 \pm 0.73\end{array}$ \\
\hline \multicolumn{13}{|l|}{ Metal: } \\
\hline $\begin{array}{l}n \\
\mathrm{Fe} \\
\mathrm{Pd} \\
\text { Total }\end{array}$ & $\begin{array}{l}14 \\
0.71 \pm 0.17 \\
99.5 \pm 0.9 \\
100.2 \pm 0.9\end{array}$ & $\begin{array}{l}13 \\
4.08 \pm 0.54 \\
95.9 \pm 1.8 \\
100.0 \pm 1.7\end{array}$ & $\begin{array}{l}9 \\
4.56 \pm 1.28 \\
95.6 \pm 2.1 \\
100.2 \pm 1.4\end{array}$ & $\begin{array}{l}7 \\
0.04 \pm 0.03 \\
99.4 \pm 1.2 \\
99.5 \pm 1.2\end{array}$ & $\begin{array}{l}7 \\
0.14 \pm 0.06 \\
99.3 \pm 1.5 \\
99.4 \pm 1.6\end{array}$ & $\begin{array}{l}2 \\
0.04 \pm 0.03 \\
99.9 \pm 2.1 \\
99.9 \pm 2.1\end{array}$ & $\begin{array}{l}10 \\
4.22 \pm 0.24 \\
96.4 \pm 1.9 \\
100.6 \pm 2.0\end{array}$ & $\begin{array}{l}9 \\
6.75 \pm 0.37 \\
92.4 \pm 1.2 \\
99.1 \pm 1.3\end{array}$ & $\begin{array}{l}6 \\
7.54 \pm 0.39 \\
91.8 \pm 1.3 \\
99.3 \pm 1.1\end{array}$ & $\begin{array}{l}6 \\
9.14 \pm 0.21 \\
90.8 \pm 0.9 \\
100.0 \pm 1.1\end{array}$ & $\begin{array}{l}9 \\
9.56 \pm 0.34 \\
90.2 \pm 1.0 \\
99.8 \pm 1.1\end{array}$ & $\begin{array}{l}9 \\
8.55 \pm 0.39 \\
90.4 \pm 1.7 \\
99.0 \pm 1.8\end{array}$ \\
\hline$a_{\mathrm{Pd}}^{\text {alloy d }}$ & $0.99 \pm 0.01$ & $0.91 \pm 0.03$ & $0.90 \pm 0.04$ & $1.00 \pm 0.02$ & $1.00 \pm 0.02$ & $1.00 \pm 0.03$ & $0.90 \pm 0.02$ & $0.84 \pm 0.02$ & $0.82 \pm 0.02$ & $0.77 \pm 0.01$ & $0.76 \pm 0.01$ & $0.79 \pm 0.02$ \\
\hline $2 \log \left(X_{\mathrm{FeO}}^{\text {glass }} / X_{\mathrm{Fe}}^{\text {alloy }}\right)$ & $1.17 \pm 0.22$ & $-1.53 \pm 0.20$ & $-2.01 \pm 0.29$ & $3.00 \pm 0.83$ & $2.39 \pm 0.36$ & $3.38 \pm 0.73$ & $-1.03 \pm 0.08$ & $-0.49 \pm 0.06$ & $-0.33 \pm 0.05$ & $0.19 \pm 0.03$ & $0.34 \pm 0.03$ & $0.00 \pm 0.05$ \\
\hline
\end{tabular}

$n=$ number of analyses.

All quoted uncertainties are $2 \sigma$ standard deviation.

${ }^{\mathrm{a}}$ Reversed experiments.

b Calculated according to the formalism given by Kilinc et al. (1983).

${ }^{c}$ Measured Pd concentrations corrected to unit Pd activity in the metal phase.

${ }^{\mathrm{d}}$ Calculated from the activity-composition relations given by Tomiska (1989). 


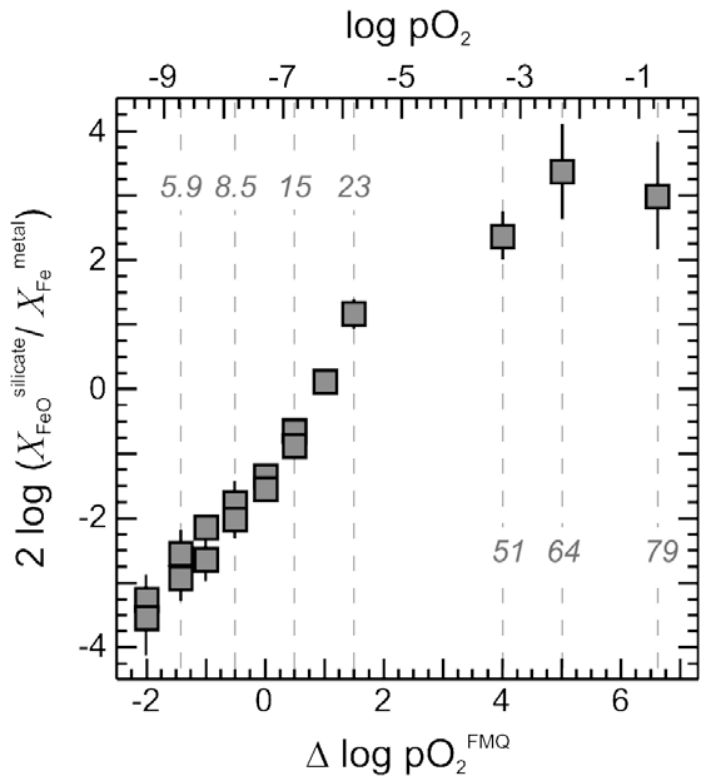

Fig. 3. Redox exchange of $\mathrm{Fe}$ in metal and $\mathrm{FeO}$ in the silicate melt versus relative $\mathrm{pO}_{2}$ (see text). Vertical dotted lines are $\left(\mathrm{Fe}^{3+} /\right.$ $\Sigma \mathrm{Fe})^{*} 100$ isopleths calculated with the formalism of Kilinc et al. (1983). Note the gradual change in slope around FMQ. Above $\mathrm{FMQ}+4, \mathrm{Fe}$ in metal phase is smaller than $0.05 \mathrm{wt} . \%$ and variations in $2 \log \left(X_{\mathrm{FeO}}^{\text {silicate }} / X_{\mathrm{Fe}}^{\mathrm{metal}}\right)$ are within the $2 \sigma$ errors.

compositional parameter, i.e. $2 \log \left(X_{\mathrm{FeO}}^{\text {silicate }} / X_{\mathrm{Fe}}^{\text {metal }}\right)$ chosen is derived from the iron-wüstite (IW) equilibrium, i.e.

$2 \mathrm{Fe}+\mathrm{O}_{2} \Longleftrightarrow 2 \mathrm{FeO}$

and variations in $2 \log \left(X_{\mathrm{FeO}}^{\text {silicate }} / X_{\mathrm{Fe}}^{\text {metal }}\right)$ are shown versus experimental $\mathrm{pO}_{2}$ (Fig. 3). The fraction of ferrous iron $(\mathrm{FeO})$ in the silicate melt is calculated using the formalism given by Kilinc et al. (1983), however, in order to illustrate as to how much iron-oxide may be present as ferric iron $\left(\mathrm{FeO}_{1.5}\right)$, the diagram is contoured with respect to molar $\left(\mathrm{Fe}^{3+} / \Sigma \mathrm{Fe}\right)^{*} 100$ isopleths.

In the $\mathrm{pO}_{2}$ range covered (FMQ-2 to FMQ+6.6), $2 \log \left(X_{\mathrm{FeO}}^{\text {silicate }} / X_{\mathrm{Fe}}^{\text {metal }}\right)$ varies by eight orders of magnitude. At a relative $\mathrm{pO}_{2}$ of FMQ (Fig. 3) we note a gradual change in slope in $2 \log \left(X_{\mathrm{FeO}}^{\text {silicate }} / X_{\mathrm{Fe}}^{\text {metal }}\right)$. This may indicate that above FMQ, $X_{\mathrm{FeO}_{1.5}}$ increases with relative $\mathrm{pO}_{2}$ at a rate faster than in the reduced region below FMQ, as predicted by the Kilinc et al. (1983) formalism. In the highly oxidized region above $\mathrm{FMQ}+4$, the $\mathrm{Pd}$ metal phase is practically $\mathrm{Fe}$ free $(<0.05 \mathrm{wt} . \%)$. Therefore, uncertainties in $2 \log \left(X_{\mathrm{FeO}}^{\text {silicate }} / X_{\mathrm{Fe}}^{\mathrm{metal}}\right)$ become substantial and any possible variations in $2 \log \left(X_{\mathrm{FeO}}^{\text {silicate }} / X_{\mathrm{Fe}}^{\text {metal }}\right)$ in that $\mathrm{pO}_{2}$ range are within the error bars.

\subsection{Metal-silicate melt equilibria with palladium}

Palladium concentrations in the glass analyzed by LAICP-MS are shown in Fig. 4A. For comparison we also show the Pd solubilities in AnDi eutectic melt (Borisov et al., 1994; Fig. 4B). As predicted by Eq. (1) and demonstrated by Borisov et al. (1994), Pd concentrations increase with increasing relative $\mathrm{pO}_{2}$, in the picrite from $1.07 \pm 0.26 \mathrm{ppm}$ (FMQ-2) to $306 \pm 19 \mathrm{ppm}$ (FMQ+6.6). All concentrations reported here are corrected to unit activity of $\mathrm{Pd}$ in the metal phase. Activity coefficients for Pd in the metal phase were calculated by fitting the molar excess Gibbs energies $\left(\Delta G^{\text {excess }}\right)$ obtained at $1292^{\circ} \mathrm{C}$ by Tomiska (1989) to the formalism of asymmetric solid solution (Thompson, 1967)

$$
\Delta G^{\text {excess }}=X_{\mathrm{Pd}} * X_{\mathrm{Fe}} *\left(W_{\mathrm{Pd}}^{G} * X_{\mathrm{Fe}}+W_{\mathrm{Fe}}^{G} * X_{\mathrm{Pd}}\right)
$$
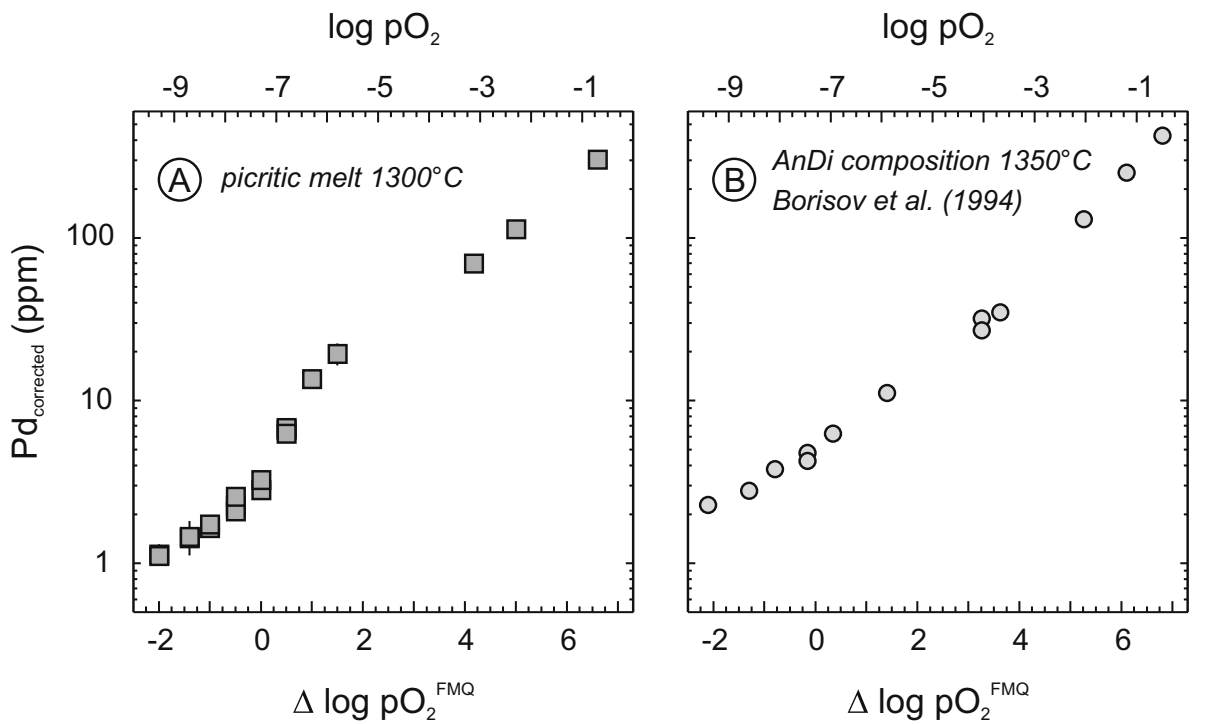

Fig. 4. (A) Variations in $\mathrm{Pd}^{n+}$ concentration in picrite versus relative $\mathrm{pO}_{2}$; all concentrations corrected to unit Pd activity in the metal phase (cf. Eq. (6)). Note the change in slope at the same relative $\mathrm{pO}_{2}$ as in Fig. 3 (see Eq. (7) and text). Error bars are $2 \sigma$, however, most errors are smaller than symbol size. Multiple data points at constant $\mathrm{pO}_{2}$ represent reversed and/or duplicated experiments (cf. Table 1). (B) Palladium solubilities in iron-free AnDi melt at $1350{ }^{\circ} \mathrm{C}$ as reported by Borisov et al. (1994) are shown for comparison (reported $1 \sigma$ uncertainties are $10 \%$ below $10 \mathrm{ppm} \mathrm{Pd}$, and 5\% above $10 \mathrm{ppm} \mathrm{Pd}$ ). 
The Margules interaction parameters obtained with this procedure are $W_{\mathrm{Pd}}^{G}=-14700 \mathrm{~J} / \mathrm{mol}$ and $W_{\mathrm{Fe}}^{G}=-30450 \mathrm{~J} / \mathrm{mol}$, respectively. The activity coefficients for $\mathrm{Fe}$ and $\mathrm{Pd}$ can then be derived from

$$
R T \ln \gamma_{\mathrm{Pd}}=X_{\mathrm{Fe}}^{2} *\left[-W_{\mathrm{Pd}}^{G}+2 * X_{\mathrm{Pd}} *\left(-W_{\mathrm{Fe}}^{G}+W_{\mathrm{Pd}}^{G}\right)\right]
$$

Between FMQ-2 and FMQ (Fig. 4A), Pd concentrations define a slope in log-concentration - $\log \mathrm{pO}_{2}$ space of $\sim 0.22 \pm 0.03(2 \sigma)$ which according to theory (i.e. Eq. (3)) would be consistent with $\mathrm{Pd}$ being present predominantly as a monovalent cation (i.e., $\mathrm{Pd}^{1+}$ or formal $\mathrm{Pd}_{2} \mathrm{O}$ species). Borisov et al. (1994), in contrast, found in that low $\mathrm{pO}_{2}$ segment a shallower slope of only $0.17 \pm 0.01$. These authors hypothesized that below FMQ, neutral species $\left(\mathrm{Pd}^{\circ}\right)$ may become important in conjunction with $\mathrm{Pd}^{1+}$. Interestingly, in that $\mathrm{pO}_{2}$ range the concentrations measured by Borisov et al. (1994) are also higher than ours by a factor of two. We suspect, therefore, that the alleged neutral $\left(\mathrm{Pd}^{\circ}\right)$ species is an artifact, i.e. a Pd component present as metallic nanonuggets or even clusters (Tredoux et al., 1995) that is indistinguishable from $\mathrm{Pd}^{n+}$ in solution if charges are analyzed by INAA.

Above FMQ, the slope of the solubility profile steepens to $\sim 0.56 \pm 0.10$ (Fig. $4 \mathrm{~A}$ ). From FMQ to $\sim \mathrm{FMQ}+2$, the dominant $\mathrm{Pd}$ oxidation state appears to be $\mathrm{Pd}^{2+}$ and the dominant oxide species PdO. Note that the change in oxidation state occurs within the same relative $\mathrm{pO}_{2}$ range where $2 \log \left(X_{\mathrm{FeO}}^{\text {silicate }} / X_{\mathrm{Fe}}^{\text {metal }}\right)$ increases (cf. Fig. 3). We suggest that around FMQ, redox exchange equilibria with major metal oxides such as

$\mathrm{Pd}_{2} \mathrm{O}^{\text {silicate }}+2 \mathrm{FeO}_{1.5}^{\text {silicate }} \Longleftrightarrow 2 \mathrm{PdO}^{\text {silicate }}+2 \mathrm{FeO}^{\text {silicate }}$

gain increasing importance, explaining why the change in $\mathrm{Pd}^{n+}$ slope coincides so closely with that of $2 \log \left(X_{\mathrm{FeO}}^{\text {silicate }} / X_{\mathrm{Fe}}^{\text {metal }}\right)$. Evidently, the effect of iron on $\mathrm{Pd}$ (and possibly HSE) solubility in silicate goes well beyond the activity effect on the metal phase identified by Borisov and Palme (2000). Consequently, above FMQ+0.5, our $\mathrm{Pd}$ concentrations in picritic melt are well above those reported by Borisov et al. (1994) in AnDi melts.

With further oxidation up to FMQ+6.6, Pd concentrations increase further but not at the same rate as in the lower $\mathrm{pO}_{2}$ segment between FMQ and FMQ+2 (Fig 4). The same is observed with respect to iron partitioning between metal and silicate (Fig. 3). We are hesitant, however, to derive an oxidation state of the dominant $\mathrm{Pd}$ cationic species in solution based on that slope (i.e., $0.26 \pm 0.04$ ) because this would imply a reduction back to $\mathrm{Pd}^{1+}$ even though relative $\mathrm{pO}_{2}$ increases. Rather, we suspect that at high $\mathrm{pO}_{2}$ the assumption of ideal behaviour of Pd in silicate melt, implicit in Eq. (1), may no longer be valid, and that we see the activity of $\mathrm{Pd}^{n+}$ increasing at a rate faster than $\mathrm{Pd}^{n+}$ solubility. Mysen et al. (1982) showed that $\mathrm{Fe}^{3+}$ tends to polymerize silicate melts, and Hillgren et al. (1996) demonstrated experimentally that solubilities of transition metals tend to decrease when silicate melts polymerize. Hence, for the highly oxidized segment in Fig. 4 we refrain from estimating an oxidation state of $\mathrm{Pd}^{n+}$ in the silicate melt, but instead attribute the change in slope to changing activity-composition relations imposed by polymerization.

To verify if Eq. (7) is valid, several more experiments were performed at constant temperature and $\mathrm{pO}_{2}$ $\left(1300^{\circ} \mathrm{C}\right.$ and $\left.\mathrm{FMQ}+0.5\right)$ with variable $\mathrm{FeO}$. The silicate matrix again was the picritic basalt, this time doped with extra $\mathrm{FeO}$ from 3 to $25 \mathrm{wt} . \%$. In the corresponding $\mathrm{X}_{\mathrm{FeO}-\text { to- }}$ tal range from 0.03 to 0.17 , iron-oxide has little measurable effect on $\mathrm{Pd}$ (Fig. 5A), but above $\mathrm{X}_{\mathrm{FeO} \text {-total }}=0.17$ we note an increase by a factor of two. This could be taken to corroborate our notion expressed by Eq. (7) that $\mathrm{Pd}^{n+}$ solubility is influenced by electron exchange with $\mathrm{FeO}$ and $\mathrm{FeO}_{1.5}$. Unfortunately though, the addition of $\mathrm{FeO}$ also lowers the polymerization degree of the silicate melt here expressed as $\mathrm{NBO} / \mathrm{T}$ (i.e. non-bridging oxygens per tetrahedron; Mysen et al., 1982) from 0.7 to 1.3 (Fig. 5B). Therefore some
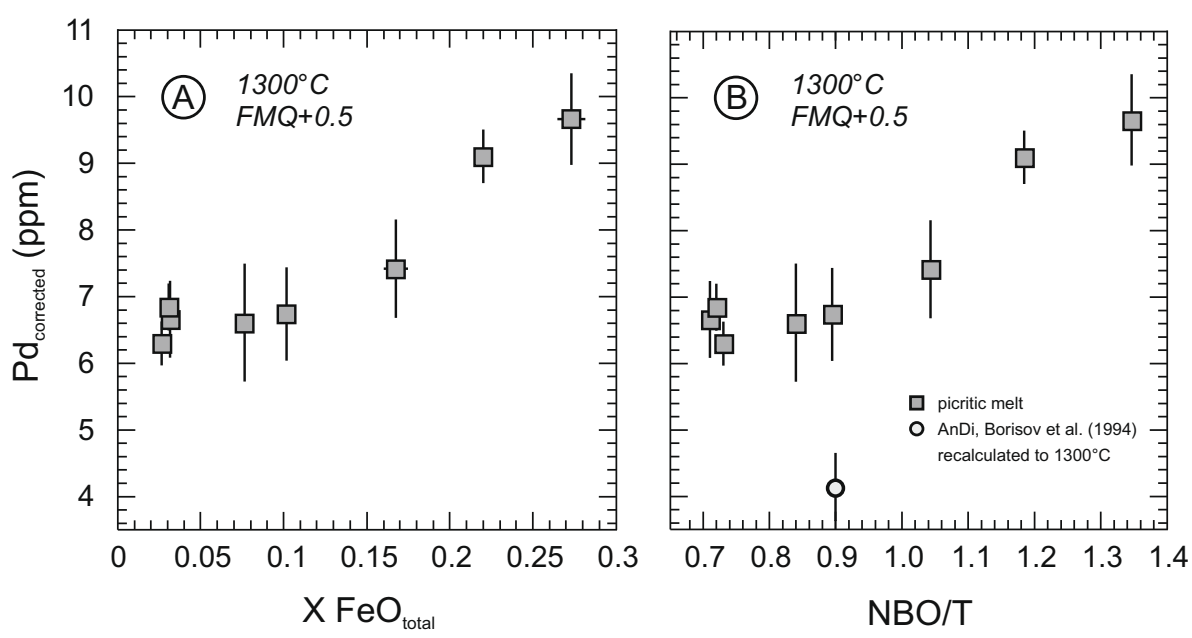

Fig. 5. (A) $\mathrm{Pd}$ concentrations at $\mathrm{FMQ}+0.5$ and $1300{ }^{\circ} \mathrm{C}$ versus $X_{\mathrm{FeO}-\text { total }}$ (as measured by EPMA; i.e. $X_{\mathrm{FeO}}+X_{\mathrm{Fe}_{2} \mathrm{O}_{3}}$ in picrite. All concentrations are corrected to unit activity of Pd in the metal phase. (B) Pd concentrations of the same set of experiments versus NBO/T (Mysen et al., 1982). The corresponding Pd concentration in $\mathrm{AnDi}\left(1300^{\circ} \mathrm{C}\right.$ and $\left.\mathrm{FMQ}+0.5\right)$ was calculated according to the equation describing Pd solubility given by Borisov et al. (1994). $2 \sigma$ errors in calculated NBO/T are smaller than symbol sizes. 
uncertainty remains as to the relative importance of the $\mathrm{pO}_{2}$ or $\mathrm{NBO} / \mathrm{T}$ contributions to Pd solubility. The crucial observation might be that $\mathrm{Pd}$ in $\mathrm{FeO}$-free AnDi melt (NBO/ $\mathrm{T}=0.9$ ) recalculated to $1300^{\circ} \mathrm{C}$, is lower by a factor of $\sim 1.5$ than in the corresponding picrite composition with $\mathrm{X}_{\mathrm{FeO} \text {-total }}=0.1$, suggesting that the $\mathrm{FeO}$ factor on Pd solubility may prevail over NBO/T.

\section{DISCUSSION AND CONCLUSIONS}

The results of this study suggest that $\mathrm{FeO}$ und $\mathrm{FeO}_{1.5}$ in silicate melts strongly influence Pd oxidation state and solubility, beyond the effect of Fe in the metal phase. In absolute terms the Pd differences between our data and the AnDi solubilities are small. However, there is a lot more detail in the picrite solubility profiles than in the AnDi solubilities, also thanks to LA-ICP-MS as tool for spatiallyresolved analysis.

From the Pd solubility profile in picritic melt (cf. Fig $4 \mathrm{~A}$ ) three distinct $\mathrm{pO}_{2}$ regions can be identified:

- Under moderately reduced conditions up to $\sim \mathrm{FMQ}$, addition of $\mathrm{FeO}$ seems to suppress $\mathrm{Pd}$ solubility (Fig. 4A and B). Presumably this is an artifact: we suggest that the presence of $\mathrm{FeO}$ (plus its equilibrium activity of $\mathrm{FeO}_{1.5}$ ) in silicate suppresses the formation of metallic Pd nano-nuggets, implying that the Pd concentrations in AnDi at low $\mathrm{pO}_{2}$ (cf. Fig. 4B) are no real solubilities but caused by metallic $\mathrm{Pd}$ nano-nuggets. If indeed neutral HSE species existed in reduced silicate melts (Borisov et al., 1994; Cottrell and Walker, 2006), which is a minority view, then the presence of $\mathrm{FeO}$ (plus some $\mathrm{FeO}_{1.5}$ ) would tend to destabilize these species relative to Pd cations (e.g. Brenan et al., 2003; Fortenfant et al., 2006; Ertel et al., 2006; Ertel et al., 2008; Yokoyama et al., 2009; Médard et al., 2010).

- In the intermediate $\mathrm{pO}_{2}$ from FMQ to FMQ+2 where most natural basalts come to lie, our solubilities in FeO-bearing melt are higher than in AnDi. In this $\mathrm{pO}_{2}$ segment, we also note a marked increase in the slope of $2 \log \left(X_{\mathrm{FeO}}^{\text {silicate }} / X_{\mathrm{Fe}}^{\text {metal }}\right)$ vs. $\mathrm{pO}_{2}$ (Fig. 3). A mechanism proposed is that in this $\mathrm{pO}_{2}$ range redox exchange equilibria such as Eq. (7) take effect. In $\mathrm{FeO}_{1.5}$ bearing silicate melts, such electron exchange equilibria stabilize a higher oxidation state of the Pd cationic species in the silicate melt relative to iron-free AnDi, because the $\mathrm{FeO}_{1.5}$ species is far more abundant than any $\mathrm{Pd}$ oxide species. The net effect of $\mathrm{FeO}-\mathrm{FeO}_{1.5}$ addition in this $\mathrm{pO}_{2}$ range is to increase $\mathrm{Pd}$ solubility (cf. Eq. (1)).

- In the high $\mathrm{pO}_{2}$ region above $\mathrm{FMQ}+2, \mathrm{Pd}$ solubilities are lower compared to the expected Pd as extrapolated from the intermediate $\mathrm{pO}_{2}$ region. Our favored explanation is that high $\mathrm{FeO}_{1.5}$ activities in this $\mathrm{pO}_{2}$ range increasingly polymerize the silicate melt, introducing marked deviations from ideal solubility and causing $\mathrm{Pd}^{n+}$ activities to increase with increasing $\mathrm{pO}_{2}$ at a rate faster than $\mathrm{Pd}^{n+}$ concentrations.

All these effects underline how important it is to supplement experiments in simple chemical systems with experi- mentation in compositionally more complex systems, for in AnDi alone none of these effects would have been evident.

The addition of $\mathrm{FeO}$ contributes toward an improved understanding in Pd solubility mechanisms in silicate melt. One should stress though that our Pd solubility data do not allow fundamentally new petrologic insights that could not have been gained as well with the AnDi dataset. No matter if we take the AnDi or the picrite Pd solubilities as our reference: all primitive melts derived from the Earth's mantle are $\mathrm{Pd}$-undersaturated. Basalts in the relative $\mathrm{pO}_{2}$ range from FMQ-1 (MORB) to FMQ+2 (IAB) typical have Pd contents around $20 \mathrm{ppb}$ (e.g. Peach et al., 1990; Woodhead et al., 2002; Bezos et al., 2005). The solubility of Pd in equilibrium with metallic $\mathrm{Pd}$ or $(\mathrm{Pd}, \mathrm{Fe})$ alloy in that $\mathrm{pO}_{2}$ range is around two orders of magnitude higher. It does not come as a surprise that neither basalts nor samples from Earth's mantle were ever reported to contain Pd-rich metallic alloys. The high solubility of $\mathrm{Pd}^{n+}$ in basaltic melt does not permit the crystallization of such phases from a melt, nor does it allow their survival during partial silicate melting in the mantle.

\section{ACKNOWLEDGMENTS}

We would like to thank M.N. Tubrett for LA-ICP-MS work at Memorial University of Newfoundland. Constructive reviews by Elizabeth Cottrell, Werner Ertel, and an anonymous reviewer helped improve this manuscript. Peter Ulmer is thanked for editorial handling. Financial support by DFG grants to Chris Ballhaus (Ba 964/24 and 26) and through a PhD scholarship of the University of Bonn to Vera Laurenz is gratefully acknowledged.

\section{REFERENCES}

Alard O., Luguet A., Pearson N. J., Griffin W. L., Lorand J.-P., Gannoun A., Burton K. W. and O'Reilly S. Y. (2005) In situ Os isotopes in abyssal peridotites bridge the isotopic gap between MORBs and their source mantle. Nature 436, 1005-1008.

Ballhaus C., Bockrath C., Wohlgemuth-Ueberwasser C., Laurenz V. and Berndt J. (2006) Fractionation of the noble metals by physical processes. Contrib. Mineral. Petrol. 152, 667-684.

Barnes S.-J., Naldrett A. J. and Gorton M. P. (1985) The origin of platinum-group elements in terrestrial magmas. Chem. Geol. 53, 303-323.

Becker H., Shirey S. B. and Carlson R. W. (2001) Effects of melt percolation on the Re-Os systematics of peridotites from a Paleozoic convergent plate margin. Earth. Planet. Sci. Lett. 188, $107-121$

Becker H., Horan M. F., Walker R. J., Gao S., Lorand J.-P. and Rudnick R. L. (2006) Highly siderophile element composition of the Earth's primitive upper mantle: constraints from new data on peridotite massifs and xenoliths. Geochim. Cosmochim. Acta 70, 4528-4550.

Bezos A., Lorand J.-P., Humler E. and Gros M. (2005) Platinumgroup element systematics in Mid-Oceanic Ridge basaltic glasses from the Pacific, Atlantic, and Indian Oceans. Geochim. Cosmochim. Acta 69, 2613-2627.

Bockrath C., Ballhaus C. and Holzheid A. (2004) Fractionation of the platinum-group-elements during mantle melting. Science 305, 1951-1953. 
Borisov A., Palme H. and Spettel B. (1994) Solubility of Pd in silicate melts: implications for core formation in the Earth. Geochim. Cosmochim. Acta 58, 705-716.

Borisov A. and Palme H. (1995) The solubility of iridium in silicate melts: new data from experiments with Ir10Pt90 alloys. Geochim. Cosmochim. Acta 59, 481-485.

Borisov A. and Palme H. (1997) Experimental determination of the solubility of platinum in silicate melts. Geochim. Cosmochim. Acta 61, 4349-4357.

Borisov A. and Palme H. (2000) Solubilities of noble metals as derived from experiments in Fe-free systems. Am. Mineral. 85, $1665-1673$.

Borisov A., Lahaye Y. and Palme H. (2004) The effect of $\mathrm{TiO}_{2}$ on $\mathrm{Pd}, \mathrm{Ni}$ and $\mathrm{Fe}$ solubilities in silicate melts. Am. Mineral. 89, 564-571.

Brandon A. D., Norman M. D., Walker R. J. and Morgan J. W. (1999) ${ }^{186} \mathrm{Os}^{-187} \mathrm{Os}$ systematics of Hawaiian picrites. Earth. Planet. Sci. Lett. 174, 25-42.

Brandon A. D. and Walker R. J. (2005) The debate over core mantle interaction. Earth. Planet. Sci. Lett. 232, 211-225.

Brenan J. M., McDonough W. F. and Dalpe C. (2003) Experimental constraints on the partitioning of rhenium and some platinum group elements between olivine and silicate melt. Earth Planet. Sci. Lett. 212, 135-150.

Capobianco C. J., Jones J. H. and Drake M. J. (1993) Metalsilicate thermochemistry at high temperature: magma oceans and "the excess siderophile element" problem of the Earth's upper mantle. J. Geophys. Res. 98, 5433-5443.

Capobianco C. J. and Hervig R. L. (1996) Solubility of Ru and Pd in silicate melts: the effect of melt composition. Lunar Planet. Sci. Conf. XXVII, 197-198, abstr.

Chase M. W. (1998) NIST-JANAF thermodynamic tables. National Institute of Standards and Technology, Washington, DC, USA.

Chou C. L. (1978) Fractionation of siderophile elements in the Earth's upper mantle. Proc. Lunar Sci. Conf. 88, 507-518.

Cottrell E. and Walker D. (2006) Constraints on core formation from Pt partitioning in mafic silicate liquids at high temperatures. Geochim. Cosmochim. Acta 70, 1565-1580.

Ertel W., O'Neill H. St. C., Sylvester P. J. and Dingwell D. B. (1999) Solubility of Pt and Rh in haplobasaltic silicate melt at $1300{ }^{\circ} \mathrm{C}$. Geochim. Cosmochim. Acta 60, 1171-1180.

Ertel W., O’Neill H. St. C., Sylvester P. J., Dingwell D. B. and Spettel B. (2001) The solubility of Rhenium in silicate melts: implications for the geochemical properties of rhenium at high temperatures. Geochim. Cosmochim. Acta 65, 2161-2170.

Ertel W., Dingwell D. B. and Sylvester P. J. (2008) Siderophile elements in silicate melts - a review of the mechanically assisted equilibration technique and the nanonugget issue. Chem. Geol. 248, 119-139.

Ertel W., Walter M. J., Drake M. J. and Sylvester P. J. (2006) Experimental study of platinum solubility in selicate melt to 14 $\mathrm{GPa}$ and $2273 \mathrm{~K}$ : Implications for accretion and core formation in Earth. Geochim. Cosmochim. Acta 70, 2591-2602.

Fonseca R. O. C., Campbell I. H., O'Neill H. St. C. and Allen C. (2009) Solubility of Pt in sulphide mattes: implications for the genesis of PGE-rich horizons in layered intrusions. Geochim. Cosmochim. Acta 73, 5764-5777.

Fortenfant S. S., Günther D., Dingwell D. B. and Rubie D. C. (2003) Temperature dependence of $\mathrm{Pt}$ and $\mathrm{Rh}$ solubilities in a haplobasaltic melt. Geochim. Cosmochim. Acta 67, 123-131.

Fortenfant S. S., Dingwell D. B., Ertel-Ingrisch W., Capmas F., Birck J. L. and Dalpe C. (2006) Oxygen fugacity dependence of Os solubility in haplobasaltic melt. Geochim. Cosmochim. Acta 70, 742-756.

Hillgren V. J., Drake M. J. and Rubie D. C. (1996) High pressure and high temperature metal-silicate partitioning of siderophile elements: the importance of silicate liquid composition. Geochim. Cosmochim. Acta 60, 2257-2263.

Holzheid A., Sylvester P., O’Neill H. St. C., Rubie D. C. and Palme H. (2000) Evidence for a late chondritic veneer in the Earth's mantle from high-pressure partitioning of palladium and platinum. Nature 406, 396-399.

Kilinc A., Carmichael I. S. E., Rivers M. L. and Sack R. O. (1983) The ferric-ferrous ratio of natural silicate liquids equilibrated in air. Contrib. Mineral. Petrol. 83, 136-140.

Kimura K., Lewis R. S. and Anders E. (1974) Distribution of gold and rhenium between nickel-iron and silicate melts: implications for the abundance of siderophile elements on the Earth and Moon. Geochim. Cosmochim. Acta 38, 683-701.

Lorand J.-P., Luguet A. and Alard O. (2008) Platinum-group elements: a new set of key tracers for the Earth's Interior. Elements 4, 247-252.

Luguet A., Pearson D. G., Nowell G. M., Dreher S. T., Coggon J. A., Spetsius Z. V. and Parman S. W. (2008) Enriched Pt-Re-Os isotope systematics in plume lavas explained by metasomatic sulfides. Science 319, 453-456.

Médard E., Schmidt M. W., Wähle M., Keller N. S. and Günther D. (2010) Pt in silicate melts: centrifuging nanonuggets to decipher core formation processes. Lunar Planet. Sci. Conf. XXXXI, \#2639, abstr.

Mysen B. O., Virgo D. and Seifert F. A. (1982) The structure of silicate melts: implications for chemical and physical properties of natural magma. Rev. Geophys. Space Phys. 20, 353-383.

O'Neill H. St. C. (1987) Quartz-fayalite-iron and quartz-fayalitemagnetite equilibria and the free energies of formation of fayalite $\left(\mathrm{Fe}_{2} \mathrm{SiO}_{4}\right)$ and magnetite $\left(\mathrm{Fe}_{3} \mathrm{O}_{4}\right)$. Am. Mineral. 72, 6775.

O'Neill H. St. C. (1991) The origin of the Moon and the early history of the Earth - A chemical model Part 2: the Earth. Geochim. Cosmochim. Acta 55, 1159-1172.

O’Neill H. St. C., Dingwell D. B., Borisov A., Spettel B. and Palme H. (1995) Experimental petrochemistry of some highly siderophile elements at high temperatures, and some implications for core formation and the mantle's early history. Chem. Geol. 120, 255-273.

O'Neill H. St. C. and Eggins S. M. (2002) The effect of melt composition on trace element partitioning: an experimental investigation on the activity coefficients of $\mathrm{FeO}, \mathrm{NiO}, \mathrm{CoO}$, $\mathrm{MoO}_{2}$ and $\mathrm{MoO}_{3}$. Chem. Geol. 186, 151-181.

Palme H. and O'Neill H. St. C. (2003) Cosmochemical estimates of mantle composition. In Treatise on geochemistry 2 (ed. R. W. Carlson). Elsevier, Amsterdam, pp. 1-38.

Peach C. L., Mathez E. A. and Keays R. R. (1990) Sulfide meltsilicate melt distribution coefficients for noble metals and other chacophile elements as deduced from MORB: implications for partial melting. Geochim. Cosmochim. Acta 54, 3379-3389.

Pearson D. G., Irvine G. J., Ionov D. A., Boyd F. R. and Dreibus G. E. (2004) Re-Os isotope systematics and platinum-group element fractionation during mantle melt extraction: a study of massif and xenolith peridotite suites. Chem. Geol. 208, 29-59.

Philipp H., Eckhardt J. D. and Puchelt H. (2001) Platinum-group elements (PGE) in basalts of the seaward-dipping reflector sequence, SE Greenland coast. J. Pet. 42, 407-432.

Puchtel I., Brandon A. D., Humayun M. and Walker R. J. (2005) Evidence for the early differentiation of the core from Pt-Re-Os isotope systematics of 2.8-Ga komatiites. Earth. Planet. Sci. Lett. 237, 118-134.

Righter K., Humayun M. and Danielson L. (2008) Partitioning of palladium at high pressures and temperatures during core formation. Nature Geosci. 1, 321-323.

Rohrbach A., Schuth S., Ballhaus C., Münker C., Matveev S. and Qopoto C. (2005) Petrological Constraints on the origin of arc 
picrites, New Georgia Group, Solomon Islands. Contrib. Mineral. Petrol. 149, 685-698.

Rose-Weston L., Brenan J. M., Fei Y., Secco R. A. and Frost D. J. (2009) Effect of pressure, temperature, and oxygen fugacity on the metal-silicate partitioning of Te, Se, and S: implications for earth differentiation. Geochim. Cosmochim. Acta 73, 4598-4614.

Schuth S., Rohrbach A., Münker C., Ballhaus C., Garbe-Schönberg D. and Qopoto C. (2004) Geochemical constraints on the petrogenesis of arc picrites and basalts, New Georgia Group, Solomon Islands. Contrib. Mineral. Petrol. 148, 288-304.

Sylvester P. J. and Eggins S. M. (1997) Analysis of Re, Au, Pd, Pt and $\mathrm{Rh}$ in NIST glass certified reference materials and natural basalt glasses by Laser Ablation ICP-MS. Geostandard. Newslett. 21, 215-229.

Thompson J. B. (1967) Thermodynamic properties of simple solutions. In Researches in geochemistry (ed. P. H. Abelson). Wiley, New York, pp. 340-361.

Tomiska J. (1989) Massenspektroskopische Ermittlung der thermodynamischen Mischungsgrößen in der kfz-Phase und Berechnung des Schmelzdiagramms Eisen-Palladium. Z. Metallkd. 80, 888-893.
Tredoux M., Lindsay N. M., Davies G. and McDonald L. (1995) The fractionation of platinum-group elements in magmatic systems with the suggestion of a novel causal mechanism. $S$. Afr. J. Geol. 98, 157-167.

Van Achterbergh E., Ryan C. G., Jackson S. E., Griffin W. L. (2001) Data reduction software for LA-ICP-MS. In Laser Ablation-ICPMS in the Earth Sciences, Principles and Applications, MAC Short Course, vol. 29 (ed. P. Sylvester). pp. 239243.

Woodhead S. J., Pearson D. G. and Thirlwall M. F. (2002) A platinum group element and Re-Os isotope investigation of siderophile element recycling in subduction zones: comparison of Grenada, Lesser Antilles arc, and the Izu-Bonin arc. $J$. Petrol. 43, 171-198.

Yokoyama T., Walker D. and Walker R. J. (2009) Low osmium solubility in silicate at high pressures and temperatures. Earth. Planet. Sci. Lett. 279, 165-173.

Associate editor: Peter Ulmer 